\title{
Transperineal ultrasound for post-prostatectomy incontinence
}

An inexpensive and noninvasive real-time imaging technique allows visualization of anatomical factors that underlie incontinence in men who have undergone radical prostatectomy.

About $10 \%$ of patients will experience problems with urinary control as a result of prostate cancer surgery. Selection of the most-effective intervention-for example, sling versus artificial sphincter-could be optimized by anatomical investigation, rather than relying solely on severity of incontinence.

To this end, a team based in Germany has conducted a pilot clinical study of transperineal ultrasound. This simple method is used widely for the diagnosis of stress incontinence in women. Ruth Kirschner-Hermanns and colleagues have now proved its feasibility in a small cohort of men with grade 2 stress incontinence that were pad-dependent in the year following prostatectomy.
The main anatomical difference between the 21 patients and 12 controls (men with excellent urinary control in the year following prostatectomy) was a greater incidence of hypermobility of the proximal urethra (defined as movement of the internal meatus $>1.5 \mathrm{~cm}$ during the Valsalva maneuver). Funneling of the bladder neck was also visualized more frequently among men with incontinence. The rate of agreement between the two investigators who assessed these features was excellent, at $94 \%$.

Perineal ultrasound also proved useful for pelvic floor training. These exercises are an important component of postprostatectomy recovery of urinary control, but many men find it difficult to perform them correctly. Allowing patients to view their real-time perineal ultrasound images helped those having difficulty to discriminate straining from voluntary contraction of pelvic floor muscles.
Images were captured in three/fourdimensional multislice mode with the patient in one of two positions. Visualization of the proximal urethra and bladder neck was optimal when the patient was supine-rather than standing-with their pelvis slightly elevated and ventrally rotated (using a cushion under the buttocks). Tilting the ultrasound probe at a $70^{\circ}$ angle after placement on the perineum between the anus and scrotum generated the best images.

Interestingly, perineal ultrasound also yielded some clues as to why surgery for incontinence correction had failed in one study participant. The position and movement of the transobturator sling that had been placed 2 months prior were easily seen during three/four-dimensional reconstruction. Suboptimal location close to the bladder neck resulted in failure to influence mobility of the urethra.

Suzanne J. Farley

Original article Kirschner-Hermanns, R. et al. Two- and three-/four dimensional perineal ultrasonography in men with urinary incontinence after radical prostatectomy. BJU Int. doi:10.1111/j.1464-410X.2011.10191.x 